\title{
Increased endocytosis in retinal vascular endothelial cells grown in high glucose medium is modulated by inhibitors of nonenzymatic glycosylation
}

\author{
A.W. Stitt, U. Chakravarthy, D. B. Archer, T. A. Gardiner \\ Department of Ophthalmology, Queen's University of Belfast, Belfast, Northern Ireland
}

\begin{abstract}
Summary We sought to determine if hyperglycaemia is responsible for increased retinal vascular endothelial-cell (RVEC) endocytosis in diabetes and to assess the role of nonenzymatic glycosylation in mediation of this novel endothelial-cell pathology. RVECs were propagated in media containing either 5 or $25 \mathrm{mmol} / \mathrm{l}$ glucose for up to 10 days after which they were exposed to the protein tracer horseradish peroxidase for $30 \mathrm{~min}$. The level of RVEC endocytosis was quantified in intact cell monolayers by electron microscopic stereology, and in cell lysates by a simple spectrophotometric method. The effect of the nonenzymatic glycosylation inhibitors, aminoguanidine and D-lysine, on high-glucose medium induced changes in RVEC endocytosis was tested by inclusion of these agents in the culture medium. RVECs exposed to $25 \mathrm{mmol} / \mathrm{l}$ glucose showed a stepwise increase in en-
\end{abstract}

docytosis of horseradish peroxidase culminating in a two- to threefold increase after 10 days. Endocytosis returned to normal levels after a further 10 days in $5 \mathrm{mmol} / 1$ glucose medium. The increase in RVEC endocytosis was markedly reduced, but not completely normalised, by aminoguanidine and D-lysine. Exposure of cultured RVECs to $25 \mathrm{mmol} / 1$ glucose causes an increase in endocytosis of similar magnitude to that experienced by RVEC in early diabetes, and implicates hyperglycaemia in the latter situation. A significant component of the increase in RVEC endocytosis appears to be mediated by nonenzymatic glycosylation. [Diabetologia (1995) 38: 1271-1275]

Key words Endocytosis, high glucose, nonenzymatic glycosylation, endothelium.
A recent study has shown a more than sixfold increase in endocytosis of horseradish peroxidase (HRP) by the endothelium of the retinal microvessels in 6-week diabetic rats, without an associated increase in capillary permeability [1]. This finding is in accord with many previous studies which have demonstrated an increased clearance of intravascular protein tracers by the retina of streptozotocin diabetic rats after similar durations of diabetes $[2,3]$. The

Received: 6 April 1995 and in revised form: 31 May 1995

Corresponding author: Dr. A. W. Stitt, Department of Ophthalmology, Queen's University of Belfast, Institute of Clinical Science, Royal Victoria Hospital, Grosvenor Road, Belfast BT12 $6 \mathrm{BA}$, Northern Ireland

Abbreviations: HRP, Horseradish peroxidase; RVEC, retinal vascular endothelial cells; DMEM, Dulbecco's modified Eagle's medium; AGE, advanced glycosylation end products. cause of increased endothelial-cell endocytosis in early diabetes is not known, but it has been speculated that increased endocytosis in retinal capillary endothelial cells may be influenced by even short periods of hyperglycaemia [1].

In view of the complexity of the diabetic milieu, it was decided to investigate the effect of glucose on endocytic activity, measured as HRP uptake, using quantitative electron microscopy in intact cells and spectrophotometric analysis of cell lysates in a simple tissue culture model of bovine retinal vascular endothelial cells (RVECs) propagated under normal and high glucose conditions. It has been suggested that nonenzymatic glycosylation of RVEC plasma membrane components could represent a specific signal for recognition and degradation of senescent molecules [4]. This could lead directly to an increase in membrane turnover. Therefore, the effect of the non- 
enzymatic glycosylation inhibitors aminoguanidine and D-lysine on increased endocytosis due to high glucose levels was also investigated in the cell-culture model.

\section{Materials and methods}

Cell culture. RVECs were isolated from cows' eyes as previously described [5]. Throughout the investigation, RVECs were grown to confluent monolayers in Dulbecco's modified Eagle's medium (DMEM) containing $20 \%$ fetal calf serum, although prior to setting up experimental conditions the cells were serum-starved for $24 \mathrm{~h}$ to ensure synchrony of their growth cycles. Under experimental conditions. RVECs were maintained in DMEM containing either 5 or $25 \mathrm{mmol} / \mathrm{lglu}$ cose and $20 \%$ fetal calf serum. The $5 \mathrm{mmol} / 1$ glucose medium osmolality was raised to that of the $25 \mathrm{mmol} / \mathrm{l}$ medium by the addition of mannitol. For all experiments, unless otherwise stated, RVECs of passage 4 to 9 (p4 to p9) were used.

Experiment (i): Estimation of endocytic activity in RVECS grown in high and low glucose medium using quantitative electron microscopy (stereology). RVECs were grown to confluency in DMEM containing $5 \mathrm{mmol} / \mathrm{l}$ glucose. After 6 days the cells were seeded into 12-well culture plates and maintained in normal or high glucose culture conditions. Following 10 days incubation, the cells in both plates were washed briefly with phosphate buffered saline and HRP (Sigma, type II, Poole, Dorset, UK) added at a concentration of $100 \mu \mathrm{g} / \mathrm{ml}$ in basal DMEM. After $30 \mathrm{~min}$ incubation at $37^{\circ} \mathrm{C}$, the cells were washed thoroughly with phosphate buffered saline and fixed for $2 \mathrm{~h}$ in situ in $2.5 \%$ glutaraldehyde in $0.1 \mathrm{~mol} / 1$ cacodylate buffer containing $10 \mathrm{mmol} / \mathrm{l}$ magnesium chloride.

Both plates were then incubated in a mixture containing diaminobenzidine and hydrogen peroxide for the electron microscopic demonstration of HRP as previously described [6]. The cells were then washed in buffer, post-fixed in $2 \%$ osmium tetroxide, dehydrated and embedded in situ in Spurr's resin.

Ultrathin transverse sections were prepared by cutting the interface of the embedding resin and the culture dish and mounted on 400-mesh copper grids. In the electron microscope, the small size of grid space in each field of view provided random selection points for cells appearing adjacent to grid bars. An image of each selected cell was transferred directly to an image analysis system and the volume density ( $v / v)$ of cell cytoplasm and HRP-filled endocytic organelles estimated using a digital stereology package (Kinetic Imaging Ltd., Liverpool, UK). A total of 70 images, from four separate wells for each treatment, was estimated blind by an investigator familiar with RVEC endosomal morphology.

Experiment (ii): Increased RVEC endocytosis: relationship to duration of high glucose exposure. Replicate 25-ml Falcon flasks were seeded at $5 \times 10^{4}$ cells/flask. One group of flasks was maintained in $5 \mathrm{mmol} / \mathrm{l}$ glucose medium while another was maintained in $25 \mathrm{mmol} / \mathrm{l}$ glucose medium. At days 4,7 and 10, a flask of cells from each group was exposed to HRP $\left(100 \mu \mathrm{g} / \mathrm{ml}\right.$ in DMEM) for $30 \mathrm{~min}$ at $37^{\circ} \mathrm{C}$, washed in phosphate buffered saline and assayed for HRP content by the spectrophotometric method outlined below.

Experiment (iii): Increased RVEC endocytosis in high glucose: modification by inhibitors of nonenzymatic glycosylation or normalisation of medium glucose concentration. RVECs were maintained in 5 and $25 \mathrm{mmol} / \mathrm{l}$ glucose for 10 days. One batch of cells from each group was propagated in medium supplemented with $5 \mathrm{mmol} / 1$ aminoguanidine while a second batch was maintained in medium containing $25 \mathrm{mmol} / 1 \mathrm{D}-l y s i n e$. The remaining flask in each group was maintained as a 5 or $25 \mathrm{mmol} / 1$ glucose control.

In a second experiment, RVECs which had been propagated in $25 \mathrm{mmol} / \mathrm{l}$ glucose for 10 days, were divided into two groups prior to a further 10 days in culture. One group was transferred to $5 \mathrm{mmol} / \mathrm{l}$ glucose medium for the extra 10 days, while the second remained in $25 \mathrm{mmol} / \mathrm{l}$ glucose during that period (20 days).

At the end of each regimen described in this section the cells were exposed to HRP ( $100 \mu \mathrm{g} / \mathrm{ml})$ for $30 \mathrm{~min}$ after which they were washed and assayed for endocytosed HRP as outlined below.

A minimum of nine replicate experiments were performed for each condition. It was ensured that at least three experiments were carried out on RVECs derived from any one isolation.

Spectrophotometric assay for endocytosed HRP in RVECs. All cells for HRP spectrophotometric assay were washed thoroughly in situ with phosphate buffered saline, detached by trypsinisation, washed again, pelleted, and resuspended in $1 \mathrm{ml}$ of citrate-phosphate buffer ( $\mathrm{pH}$ 5.0). At this point, a 100$\mu$ l sample was taken from each cell suspension, stained with trypan blue and the cell density and viability estimated using a haemocytometer. The remaining cells were then subjected to ultrasonic disruption for $30 \mathrm{~s}$ and centrifuged to leave a cell lysate containing previously intracellular HRP. For spectrophotometric assay, $50-\mu l$ samples of this lysate, together with $50 \mu 1$ of citrate-phosphate buffer containing o-phenylenediamine $4 \mathrm{mmol} / \mathrm{l}$ and $0.004 \% \mathrm{H}_{2} \mathrm{O}_{2}$ were pipetted onto a 96-well ELISA plate. The plate was then gently agitated in darkness at room temperature and the reaction stopped after $1 \mathrm{~h}$ with $0.2 \mathrm{~mol} / 1$ $\mathrm{H}_{2} \mathrm{SO}_{4}$. When $o$-phenylenediamine is employed as hydrogen donor, HRP yields a soluble brown reaction product, the optical density of which may be read using a multiplate reader at $492 \mathrm{~nm}$. Before comparison of HRP activity between test and control cells, the results for each batch were corrected for cell number according to the haemocytometer counts obtained above. Unless stated otherwise, all drugs and reagents were obtained from the Sigma Chemical Company.

\section{Statistical analysis}

Statistical analysis was performed using the Wilcoxon rank sum test.

\section{Results}

Experiment (i) Qualitative results. Electron-dense HRP reaction product was observed in the endosomal compartment of the RVECs and qualitatively there appeared to be a greater density in cells grown in $25 \mathrm{mmol} / 1$ glucose compared to those grown in $5 \mathrm{mmol} / \mathrm{l}$. The endosomal compartments containing HRP (Fig. 1) included small vesicular or tubular structures (120-150 nm diameter), larger vacuoles in which the HRP reaction product was confined to the 


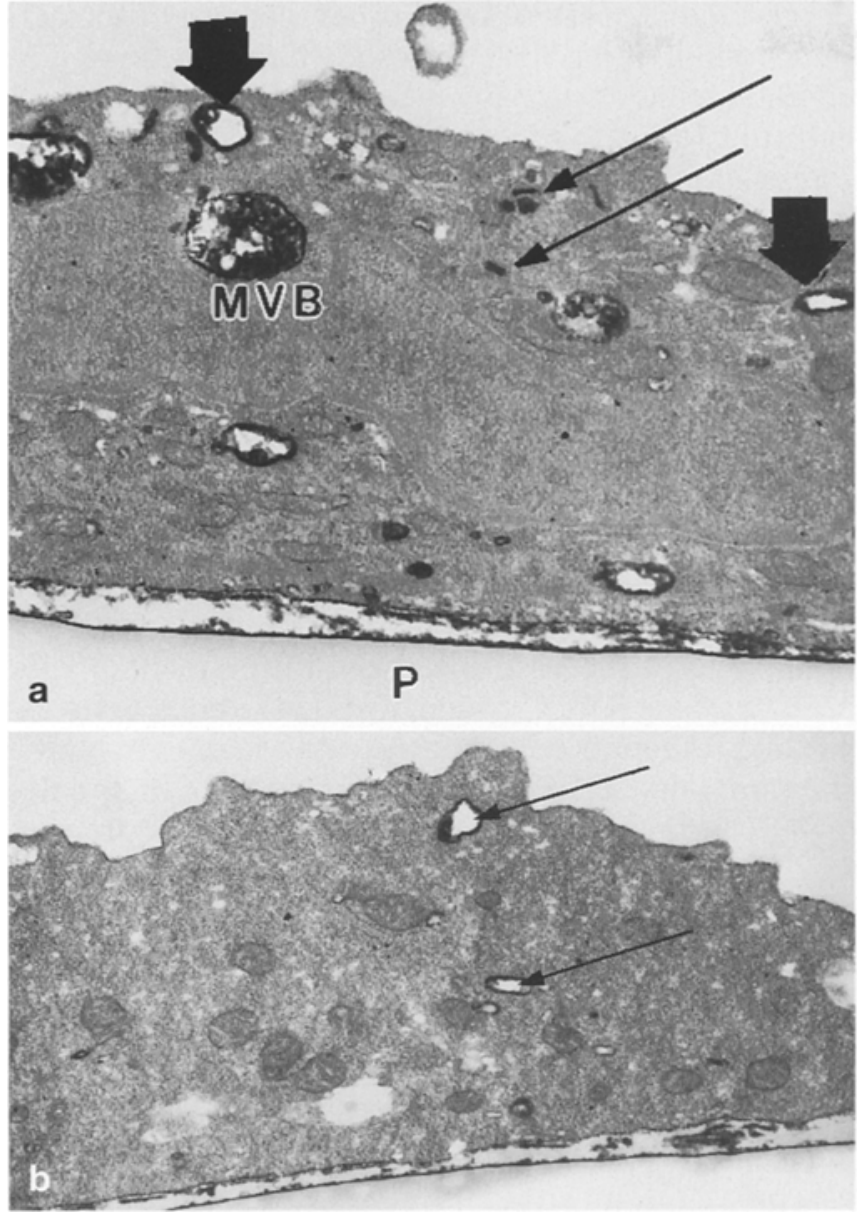

Fig. 1a. Electron micrograph of a retinal vascular endothelial cell (RVEC) following 10 days of culture in $25 \mathrm{mmol} / 1$ glucose medium, and $30 \mathrm{~min}$ exposure to horseradish peroxidase (HRP). HRP reaction product is present in small vesicular/tubular structures (small arrows), larger endosomal vacuoles (large arrows), and large multivesicular bodies (MVB). P; plastic of culture dish. b. Electron micrograph of RVEC following 10 days' culture in $5 \mathrm{mmol} / 1$ glucose medium and $30 \mathrm{~min}$ exposure to HRP. HRP containing endosomes (arrows). Magnification X 16,000

internal surface of the limiting membrane (300$350 \mathrm{~nm}$ diameter), and large multivesicular bodies (500-600 $\mathrm{nm}$ diameter).

Experiment (i) Quantitative results. Stereological analysis (Fig. 2) demonstrated that after 10 days under test conditions the level of RVEC endocytosis, as measured by the volume fraction of HRP-containing endosomes, was $380 \%$ greater in cells exposed to $25 \mathrm{mmol} / \mathrm{l}$ glucose than in those maintained in $5 \mathrm{mmol} / 1$ glucose $(p<0.001)$.

Experiment (ii). RVEC exposed to either normal $(5 \mathrm{mmol} / \mathrm{l})$ or high $(25 \mathrm{mmol} / \mathrm{l})$ ambient glucose levels did not show any difference in cell viability which was generally over $95 \%$. Cells cultured under high glucose conditions for 4 days revealed a small, but

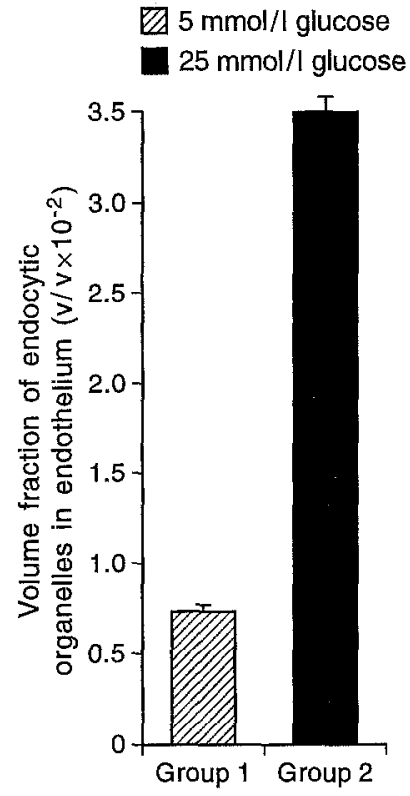

Fig.2. Stereological estimation of RVEC endocytosis following exposure to high glucose medium. The volume fraction $(\mathrm{v} / \mathrm{v})$ of endocytic organelles containing HRP reaction product is shown for $5 \mathrm{mmol} / \mathrm{lglucose}$ (group 1) and after 10 days exposure to $25 \mathrm{mmol} / \mathrm{l}$ glucose (group 2). Results are expressed as $\mathrm{v} / \mathrm{v} \times 10^{-2} \pm \mathrm{SEM}$

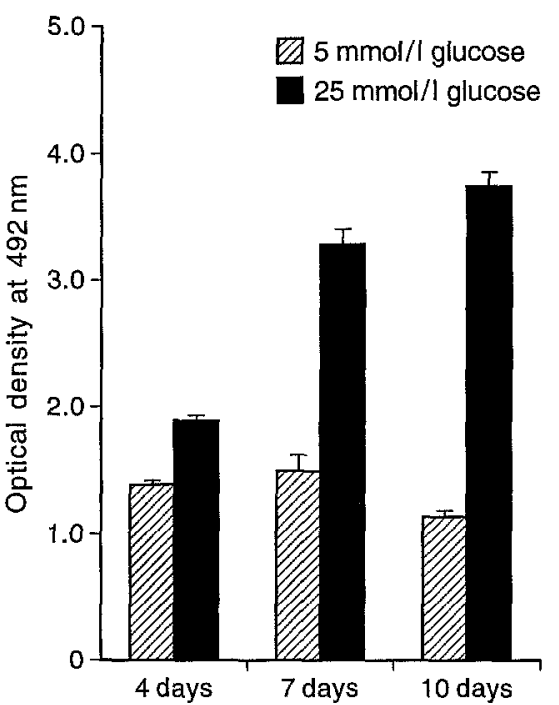

Fig. 3. Spectrophotometric estimation of RVEC endocytosis after exposure to high glucose medium for 4,7 and 10 days, and HRP for $30 \mathrm{~min}$. The vertical axis shows the optical density obtained at $492 \mathrm{~nm}$, following incubation of cell lysate with o-phenylenediamine, and represents a function of the activity of internalised HRP. Error bars represent \pm SEM

statistically insignificant increase in RVEC endocytosis as measured by the relative activity of internalised HRP in the spectrophotometric assay (Fig. 3). However, after 7 days, endocytosis in the $25 \mathrm{mmol} / \mathrm{l} \mathrm{glu}-$ cose cells was found to be $120 \%$ over that of the $5 \mathrm{mmol} / 1$ glucose cells $(p<0.002)$ and this difference increased to $230 \%$ after 10 days $(p<0.001)$.

Experiment (iii). Aminoguanidine and D-lysine reduced the high glucose-induced increase in RVEC endocytosis to +90 and $+44 \%$ of the respective $5 \mathrm{mmol} / \mathrm{l}$ glucose controls $(p<0.001)$ (Fig. 4$)$. Neither of these agents had a significant influence on en- 


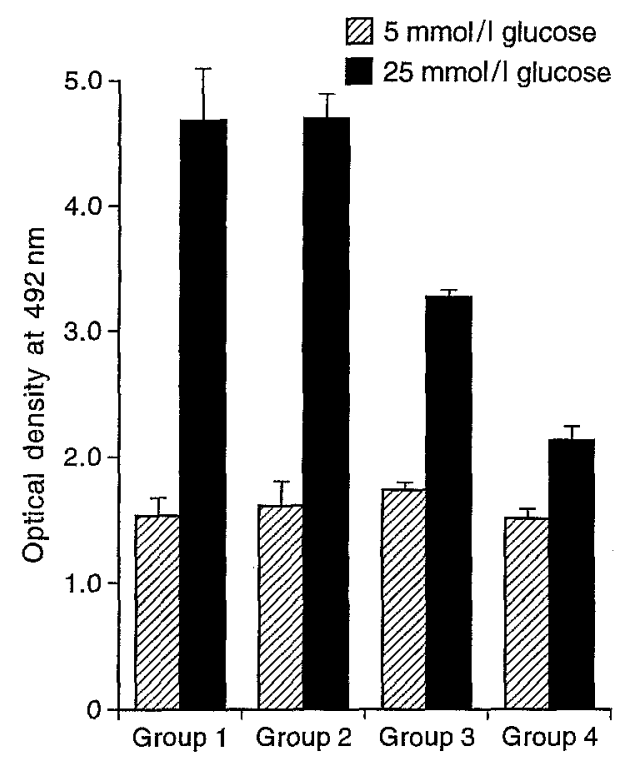

Fig. 4. Spectrophotometric estimation of RVEC endocytosis following exposure to high glucose: effect of inhibitors of NEG or normalisation of medium glucose. Group 1 shows endocytosis in RVECs grown in 5 vs $25 \mathrm{mmol} / \mathrm{l}$ glucose for 10 days. Group 2 same as group 1, except that both sets of cells were pre-exposed to $25 \mathrm{mmol} / \mathrm{l}$ glucose for 10 days. Note that endocytosis in the $5 \mathrm{mmol} / \mathrm{l}$ RVECs approximates to the $5 \mathrm{mmol} / \mathrm{l}$ cells of group 1 , in spite of pre-incubation in high glucose. Groups 3 and 4 show a significant reduction of endocytosis in the high glucose group by $5 \mathrm{mmol} / \mathrm{l}$ aminoguanidine and $25 \mathrm{mmol} / \mathrm{l} \mathrm{D}$-lysine, respectively. Error bars represent $\pm \mathrm{SEM}$

docytosis in RVECs maintained in $5 \mathrm{mmol} / \mathrm{l}$ glucose (Fig. 4).

Endocytic activity in RVECs (returned to $5 \mathrm{mmol} / \mathrm{l}$ glucose for 10 days following 10 days in $25 \mathrm{mmol} / \mathrm{l}$ glucose) was not significantly different from cells which had never undergone elevated glucose concentrations (Fig. 4). RVECs which were exposed to continuing high ambient glucose levels (20 days) did not show any further increase in endocytosis when compared to cells exposed to such conditions for 10 days (Fig. 4).

\section{Discussion}

This study has used a simple spectrophotometric assay on lysates of cultured RVECs reflecting the degree of endocytic activity under different conditions. The complementary morphological technique used confirmed the internalisation of the tracer by the endosomal system of the cultured RVECs and strongly correlated with the degree of endocytosis as assessed by spectrophotometry.

The enormous increase in RVEC endocytosis in response to high glucose levels in the present study strongly suggests that the similarly exaggerated endocytic activity in RVECs during early streptozotocin diabetes in rats [1] occurs as a consequence of hy- perglycaemia, and not some other element of the diabetic milieu. It has also been shown that the high glucose-associated increase in endocytosis observed in cultured RVECs is significantly reduced by two different inhibitors of nonenzymatic glycosylation, and normalised by returning the cells to low glucose-containing media.

The various endosomal compartments showing internalised HRP in the present study were similar in structure to those previously described in cultured RVECs [5] and other non-vascular-cell types [7, 8]. However, as HRP acts as a marker of "fluid-phase", adsorptive, and receptor-mediated endocytosis [1], it is not possible to identify which component of plasma membrane internalisation is chiefly affected by exposure of RVECs to high glucose media.

In spite of the vast difference in the technologies used to assess RVEC endocytosis in the current investigation, the markedly greater increase in the volume fraction of HRP-stained endocytic organelles $(380 \%)$ as measured by electron microscope stereology, compared to the increase in the enzymatic activity of endocytosed HRP ( $230 \%$ ) in the spectrophotometric assay, deserves some comment. A direct comparison of the ratios of endocytosis obtained from the two assays would have to assume the unlikely situation of an equal concentration of HRP being present throughout all the endocytic compartments visualised in the electron microscope study. Also, as HRP was only present within the glycocalyx on the internal surface of many of the endosomes, much of the measured volume fraction does not contain HRP. Therefore, cells with more endosomes (RVECs in $25 \mathrm{mmol} / \mathrm{l}$ glucose) will contain a greater proportion of "empty" endosome volume fraction than cells with fewer endocytic vacuoles (RVECs in $5 \mathrm{mmol} / 1$ glucose).

High glucose levels have been shown to increase nonenzymatic glycosylation, the products of which have been implicated in a wide variety of pathological mechanisms associated with diabetes and aging $[9,10]$. The initial stages of protein glycation are reversible and the early intermediates appear to be less important in the complications of diabetes than advanced glycosylation end products (AGEs). AGEs induce more significant protein modification than early glycation products [9-11]. It was, therefore, of interest that in the current investigation, the amino acid D-lysine, a competitive inhibitor of early nonenzymatic glycosylation $[12,13]$, produced a more profound reduction in RVEC endocytosis than aminoguanidine. However, both can inhibit the distal AGE product formation and both may interact with the same AGE intermediate, following the formation of an Amadori product [14]. Moreover, intracellular accumulation of AGEs in endothelial cells [15] follows a similar time-course to the increase endocytic activity described in the present study. 
It is noteworthy that the increased endocytic activity in RVEC exposed to high glucose medium for 20 days was similar to that of cells exposed for 10 days. This may reflect saturation of the mechanisms reponsible for endocytosis following 10 days of exposure to $25 \mathrm{mmol} / 1$ glucose. Normalisation of RVEC endocytosis by $5 \mathrm{mmol} / 1$ glucose following 10 days pre-exposure to $25 \mathrm{mmol} / \mathrm{l}$ glucose, may suggest that rapid and reversible membrane glycation, is of greater importance in the mediation of increased RVEC endocytosis, than the effects of long-lived AGEs. However, it has recently been shown that AGEs may influence even short-lived proteins [15] and, therefore the return to normal endocytic function (10 days) after $25 \mathrm{mmol} / \mathrm{l}$ glucose exposure may still implicate AGEs in this phenomenon. It may also be speculated that glycation damage of important structural or receptor proteins on the RVEC apical plasma membrane may be interpreted by the cell as physiological aging, thereby initiating plasma membrane recycling, a process dependent upon the endocytic pathway [16].

It is possible that even very low concentrations of AGEs within the plasma membrane may accelerate membrane recycling and that the differential in the efficacy of D-lysine and aminoguanidine may be explained by the lower medium concentration of the latter agent (concentrations of aminoguanidine higher than $5 \mathrm{mmol} / \mathrm{l}$ were found to produce significant growth inhibition in RVECs, unpublished observations). In such a scenario the normalisation of endocytosis following return of the cells to $5 \mathrm{mmol} / \mathrm{lglu}-$ cose could be explained by the removal of plasma membrane AGEs by endocytosis.

The failure of both D-lysine and aminoguanidine to reduce the high-glucose-related increase in RVEC endocytosis to normal levels, suggests that while more than one endocytosis-dependent plasma membrane function may be stimulated by high glucose levels, not all may be influenced by glycosylation. It is therefore of interest that activation of protein kinase $\mathrm{C}$, which is known to occur in vascular endothelium in diabetes [17], has been implicated in the control of both "fluid-phase" endocytosis [18] and receptormediated endocytosis in certain systems [19]. Future studies incorporating inhibition of both protein kinase $\mathrm{C}$ and nonenzymatic glycosylation, and the use of tracers which are only internalised by receptor-mediated endocytosis, should help clarify this issue.

Acknowledgements. The authors acknowledge the assistance and technical expertise of Ms. C. Hughes and Ms. P. Linton during this investigation. This work was funded by The Guide Dogs for the Blind Association (UK).

\section{References}

1. Gardiner TA, Stitt AW, Archer DB (1995) Retinal vascular endothelial cell endocytosis increases in early diabetes. Lab Invest 72: 439-444

2. Williamson JR, Chang K, Tilton RG et al. (1987) Increased vascular permeability in spontaneously diabetic $\mathrm{BB} / \mathrm{W}$ rats and in rats with mild versus severe streptozotocin-induced diabetes. Diabetes 36: 813-821

3. Pugliese G, Tilton RG, Speedy A et al. (1989) Effects of very mild versus overt diabetes on vascular haemodynamics and barrier function in rats. Diabetologia 32: 845-857

4. Vlassara H, Brownlee M, Cerami A (1985) High-affinity receptor mediated uptake and degradation of glucosemodified proteins: a potential mechanism for the removal of senescent macromolecules. Proc Natl Acad Sci USA 82: 5588-5592

5. Stitt AW, Anderson HR, Gardiner TA, Bailie JR, Archer DB (1994) Receptor mediated endocytosis and intracellular trafficking of insulin and LDL in retinal microvascular endothelial cells. Invest Ophthal Vis Sci 35: 3384-3392

6. Gardiner TA, Archer DB (1986) Endocytosis in the retinal and choroidal microcirculation. Brit J Ophthalmol 70: 361372

7. Helenius A, Mellman I, Wall D, Hubbard A (1983) Endocytosis. Ann Rev Biochem 46: 699-722

8. De Bruyn PPH, Michelson S, Bankston PW (1985) In vivo endocytosis by bristle-coated pits and intracellular transport of endogenous albumin in the endothelium of the sinuses of liver and bone marrow. Cell Tissue Res 240: 1-7

9. Brownlee M (1992) Glycation products and the pathogenesis of diabetic complications. Diabetes Care 15: 1835-1843

10. Vlassara H, Bucala R, Striker L (1994) Pathogenic effects of advanced glycosylation: biochemical, biologic, and clinical implications for diabetes and aging. Lab Invest 70 (2): 138-151

11. Brownlee M, Cerami A, Vlassara H (1988) Advanced glycosylation end products in tissue and the biochemical basis of diabetic complications. New Eng J Med 318: 1315-1321

12. Sensi M, De Rossi MG, Celi FS et al. (1993) D-lysine reduces the non-enzymatic glycation of proteins in experimental diabetes mellitus in rats. Diabetologia 36: 797-801

13. Sensi M, Pricci F, De Rossi MG, Morano S, Di Mario U (1993) D-lysine effectively decreases the nonenzymatic glycation of proteins in vitro. Clin Chem 35: 384-387

14. Edelstein D, Browalee M (1992) Mechanistic studies of advanced glycosylation end product inhibition by aminoguanidine. Diabetes 41: 26-28

15. Giardino I, Edelstein D, Brownlee M (1994) Nonenzymatic glycosylation in vitro and in bovine endothelial cells alters basic fibroblast growth factor activity. J Clin Invest 94: $110-117$

16. Steinman RM, Mellman IS, Muller WA, Cohn ZA (1983) Endocytosis and the recycling of plasma membrane. J Cell Biol 96: 1-27

17. De Rubertis FR, Craven PA (1994) Activation of protein kinase $\mathrm{C}$ in glomerular cells in diabetes. Mechanisms and potential links to the pathogenesis of diabetic glomerulopathy. Diabetes 43: $1-8$

18. Phaire-Washington L, Wang E, Silverstein SC (1994) Phorbol myristate acetate stimulates pinocytosis and membrane spreading in mouse peritoneal macrophages. J Cell Biol 86: 634-640

19. Backer JM, King GL (1991) Regulation of receptor mediated endocytosis by phorbol esters. Biochem Pharmacol 41(9): 1267-1277 